\title{
SEMIGROUPS WITH IDENTITY ON A MANIFOLD $\left({ }^{1}\right)$
}

\author{
BY \\ PAUL S. MOSTERT AND ALLEN L. SHIELDS
}

The principal question with which this paper is concerned is the following: if a manifold $M$ admits a continuous associative multiplication with identity and no other idempotents, is it a group? We are able to show that in the case $M$ is the line or plane, the answer is in the affirmative. However, the general problem remains open.

We show that in any case, there is a maximal, connected, open subgroup $G$ of $M$ containing the identity. This then determines a subspace $L$, the boundary of $G$ in $M$, which we show is an ideal of $G^{-}$(the closure of $G$ ). Thus, a study of $M$ necessarily involves a thorough study of $L$. We conjecture, for example, that $L$ always contains an idempotent (we prove this in case $L$ is the line or plane, or when $M$ is two dimensional and $L$ is a regular boundary). Clearly this would imply an affirmative answer to the question raised above for arbitrary dimensions.

The existence of the open subgroup $G$ gives us as corollaries two results of A. D. Wallace $[1 ; 2]$, both of which are encompassed in the following more general result: if a compact manifold $S$ with boundary $B$ is a semigroup with identity, then the set of elements with inverses is contained in $B$ or else is all of $S$.

We consider also the following problem: if the closed right half plane is a semigroup in which the open half plane is a group $G$, how many possibilities are there for multiplication on the $y$-axis $(=L)$ ? We show that if $G$ is isomorphic to the two dimensional vector group, there are exactly four possibilities, and examples are given of each of them. If $G$ is the nonabelian group, we can identify two possibilities, but we are unable to prove these are all. (It might be interesting to note that even in the plane, we are unable to decide whether $L$ need be a regular boundary for $G$ or not.)

Background material. 0.1. By a semigroup $S$ we mean a topological semigroup, that is, a Hausdorff space with a continuous associative multiplication. If there is an identity it will be denoted by $1 . H(1)$ denotes the set of elements with inverses; it is a group.

0.2 . A set $A$ in a semigroup $S$ is a left ideal if $S A \subset A$. Similarly one defines right ideal. By ideal we mean a two-sided ideal. If $A$ is a left ideal and $B$ is a right ideal, then $B A \subset A \cap B$, so every left ideal meets every right ideal.

Presented to the Society, December 28, 1955 under the title On semigroups on a manifold; received by the editors September 26, 1957.

(1) This work was supported in part by Tulane University's National Science Foundation contract. 
The closure of an ideal is an ideal, and similarly for left and right ideals.

0.3 . In a connected semigroup $S$ with identity every ideal is connected. Indeed, assume $A$ were a disconnected ideal, and let $x, y \in A$ be in different components. Then $x S$ is a connected subset of $A$ containing $x$, and is therefore contained in the $x$-component. Similarly $S y$ is in the $y$-component. But by $0.2, x S$ meets $S y$, which is impossible.

0.4. $A^{-}$denotes the topological closure of $A ; A \backslash B$ denotes set-theoretic difference.

0.5. A compact semigroup always contains an idempotent [3].

1. 1.1. Lemma. Let $Q$ be the solid unit ball in Euclidean $n$-space, and let $f$ be a map of $Q$ into itself such that: $d(x, f(x))<1 / 2$ for all $x$. Then $0 \in f(Q)$.

This is an immediate consequence of the Brouwer fixed point theorem. See Hurewicz-Wallman, Dimension theory, Chapter 6, Example VI.2, p. 75.

1.2. Theorem. Let $S$ be a semigroup with identity having a Euclidean neighborhood $U$ of the identity. Then there is a neighborhood $V \subset U$ of 1 such that every element of $V$ has an inverse in $U . H(1)$ is an open subset of $S$ and is a Lie group.

Proof. We identify $U$ with Euclidean $n$-space with its metric, $d$. Let $\epsilon>0$ be given and let $Q$ be the solid ball of radius $\epsilon$ centered at the identity. By the uniform continuity of multiplication on $Q$ there is a $\delta$ such that

$$
d(x, x y)<\epsilon / 2, \quad d(x, y x)<\epsilon / 2
$$

whenever $d(y, 1)<\delta$ and $x \in Q$. By Lemma $1.1,1 \in Q y$ and $1 \in y Q$, so $y$ has both a left and a right inverse in $Q$, hence has a unique two-sided inverse in $Q$.

The proof shows that inversion is a continuous operation, and therefore $H(1)$ is a topological group. Since it contains an open set it must be an open set, so it is locally-Euclidean and therefore a Lie group.

1.3. CoROllary. If $S$ is a compact connected semigroup with identity having a Euclidean neighborhood of the identity, then $S$ is a Lie group.

Proof. By $1.2 H(1)$ is open, and in a compact semigroup it is always closed, hence it must be all of $S$.

1.4. The pair $(S, B)$ is called a relative manifold with boundary $B$ if $S$ is a connected Hausdorff space and $B$ is a closed subset such that $S \backslash B$ is locally Euclidean.

1.4.1. Corollary. If $S$ is a compact connected semigroup with identity and $(S, B)$ is a relative manifold with boundary, then either $S$ is a group or $H(1) \subset B$.

Proof. Assume an element $g$ of $H(1)$ is in $S \backslash B$ and thus has a Euclidean neighborhood. Then there is a Euclidean neighborhood of 1 , and by $1.3 S$ is a group. 
In case $S$ is a subset of Euclidean space and has interior points then it cannot be a group (since a group is homogeneous space) and we get the result of Wallace quoted at the beginning.

Theorem 1.2 is not true for general locally convex linear spaces. For example, if $S$ is the set of all real-valued continuous functions defined on $-\infty<x$ $<\infty$, with the (metric) topology of uniform convergence on compact subsets and with composition as the semigroup operation, then $H(1)$ is not open. This example is not a Banach space and we do not know whether or not Theorem 1.2 is true for such spaces. In particular, can Hilbert space be a semigroup with identity in which $H(1)$ is not open?

2. In this section we consider connected semigroups $S$ with identity in which $H(1)$ is open. By 1.2 this includes the case of a semigroup with identity on a manifold. It also includes, for example, the case of a commutative Banach algebra with identity.

We shall use the following notations. $G$ will denote the component of $H(1)$ containing the identity, and $L$ will be the boundary of $G$. We shall be concerned only with the subsemigroup $G^{-}=G \cup L$. We show that $L$ is an ideal in $G^{-}$, and that $L$ is connected. We conjecture that $L$ must contain an idempotent when $S$ is a manifold but we are able to prove this only with additional assumptions.

\subsection{Lemma. $L$ is a connected ideal in $G^{-}$.}

Proof. We show that $L$ is an ideal in $G^{-}$, the connectedness then follows by 0.3 . If $x \in L, g \in G$ and $x g=h \in G$, then $x=h g^{-1} \in G$ which is impossible. So $L G \subset L$. Similarly $G L \subset L$. Since $L$ is closed $L G^{-} \subset L$ and $G^{-} L \subset L$.

2.2. If $x, y \in L$, then $x G$ and $y G$ are either disjoint or identical. These are the right orbits if we think of $G$ acting as a transformation group on $L$ by right multiplication.

2.2.1. The closure of each orbit is a right ideal. Indeed, $(x G)^{-}=\left(x G^{-}\right)^{-}$, and $x G^{-}$is a right ideal (in $G^{-}$). Similarly $(G y)^{-}$is a left ideal for each $y \in L$. Hence by 0.2 each right orbit closure meets each left orbit closure.

2.2.2. If $z \in(x G)^{-}$then $z G \subset(x G)^{-}$- since $(x G)^{-}$is a right ideal.

2.3. LEMma. If $x G=G x$ for some $x \in L$, then either $x G$ is a group, or it contains none of its products, that is, $w, y \in x G$ implies $w y \in x G$.

Proof. Assume we have a pair $w, y \in x G$ such that $w y=v \in x G$. Then $v G=w G=y G=x G=G x=G y=G w=G v$. To show $w G$ is a group it suffices to show: $(w G) u=u(w G)=w G$ for each $u \in w G$. Let $u=x g=h x$ with $g, h \in G$. Then $u(w G)=x g(G y)=(x G) y=(G w) y=G v=w G$, and simila.ly for $(w G) u$.

Example 4.6.2 shows that if the hypothesis $x G=G x$ is omitted, then $G x$ may be a closed subsemigroup that is not a group. In all our examples either $x G$ is a subsemigroup or it contains none of its products, but we do not know if this is always true. 
2.4. Lemma. If there is an $x \in L$ for which $x^{2} \in x G$ and $x^{2} \in G x$ then $L$ contains an idempotent.

Proof. By hypothesis there are elements $g, h \in G$ for which $x=x^{2} g=h x^{2}$. Let $e=x g=h x^{2} g=h x$. Then $e^{2}=(h x)(x g)=e$.

2.5. Lemma. If there is a closed right orbit $x G$ and a closed left orbit $G y$, $x, y \in L$, then $L$ contains an idempotent.

Proof. By 2.2.1, $x G$ meets $G y$. Let $z \in x G \cap G y$. Then $z G=x G, G z=G y$, so $z G$ and $G z$ are subsemigroups. Thus $z^{2} \in z G \cap G z$ and the result follows from 2.4 .

2.6. For any set $A \subset L$, let $F(A)=A^{-} \backslash A$.

2.6.1. Theorem. If $L$ is finite dimensional and $\operatorname{dim} F(x G)<\operatorname{dim} x G$, $\operatorname{dim} F(G x)<\operatorname{dim} G x$ for each $x \in L$, then $L$ has an idempotent.

Proof. Let $x \in L$ be an element such that $x G$ has minimum dimension for right orbits. Then $x G$ is closed, for if $z \in F(x G)$ then $z G \subset F(x G)$ by 2.2.2 and $\operatorname{dim} z G<\operatorname{dim} x G$, which is impossible. Similarly there is a closed left orbit, and the result follows from 2.5 .

2.7. Corollary. If $S$ is the plane and $G$ is the open right half plane, then there is an idempotent on the $y$-axis.

3. In this section we show that if the line or the plane is a semigroup with identity and no other idempotents then it is a group.

3.1. THEOREv. If the real line $S$ is a semigroup with identity and no other idempotents then it is a group.

Proof. If $G$ is the identity component of $H(1)$, then by $1.2 G$ is an open interval. So it is isomorphic to the multiplicative group of positive real numbers since there is only one group on the line. Hence for $x \neq 1, x$ always comes between 1 and $x^{2}$. Now if $G \neq S$, let $e$ be an endpoint of $G$ and let $x_{n} \rightarrow e$, $x_{n} \in G$. Then $x_{n}^{2} \rightarrow c$, so $e^{2}=e$.

For the remainder of the section $S$ will denote the Euclidean plane which we assume to be a semigroup with identity and no other idempotents. We assume $S$ is not a group, and therefore, with the notations of $\$ 2, L$ is not vacuous.

3.2. $\operatorname{Dim} L=1, \operatorname{dim} x G=\operatorname{dim} G x=1$ for each $x \in L$.

Proof. $L$ is the boundary of an open set, so $\operatorname{dim} L=1$. Each $x G$ is a connected subset of $L$; if it were zero dimensional it would be a point. But then $x G=x$, which implies $x G^{-}=x$, and therefore $x^{2}=x$. Similarly, $\operatorname{dim} G x=1$.

3.3. If $G_{r}(x)=\{g \in G: x g=x\}$ and $G_{l}(x)=\{g \in G ; g x=x\}$, then $G_{r}(x)$ $=G_{l}(x)$.

Proof. Dim $G_{r}(x)=\operatorname{dim} G-\operatorname{dim} x G=1$, and similarly for $G_{l}(x)$. Hence if 
$G_{r}(x) \neq G_{l}(x)$ we must have $G_{r}(x) G_{l}(x)=G$. Thus there exist $g_{n} \in G_{r}(x)$, $h_{n} \in G_{l}(x)$ such that $g_{n} h_{n} \rightarrow x$. Hence $x^{3}=\lim x g_{n} h_{n} x=x^{2}$. Thus $\left(x^{2}\right)^{2}=x^{3} x=x^{2} x$ $=x^{3}=x^{2}$, so $L$ contains the idempotent $x^{2}$.

We shall call the group leaving $x$ fixed $G(x)$.

3.4. If $x, y \in L$, then $G(x)=G(y)$. This group will be called $H$.

Proof. As in 3.3, $\operatorname{dim} G(x)=\operatorname{dim} G(y)=1$. If they are unequal, then $G(x) G(y)=G$. Also, $x y G(y)=x y, G(x) x y=x y$, so $G(x) \cup G(y) \subset G(x y)$, and therefore $G \subset G(x y)$. But then $x y$ is a zero for $G^{-}$, and in particular is idempotent.

3.5. $H$ is a normal subgroup of $G$.

Proof. If $g \in G, x \in L$, then $g x \in L$. Hence $H g x=g x$, so that $\left(g^{-1} H g\right) x=x$. Then by $3.4 \mathrm{~g}^{-1} \mathrm{HgCH}$.

REMARK. One can easily verify that $H$ is closed in $G^{-}$, so one is tempted to form the quotient semigroup $G^{-} / H$. Unfortunately this will not in general be Hausdorff; see 4.5.5.

\section{6. $H$ is isomorphic to the group of real numbers.}

Proof. This follows from the following facts: (i) $H$ is closed in $G$; (ii) $\operatorname{dim} H$ $=1$; (iii) $G$ has no nontrivial compact subgroups; (iv) no orbit $x G$ where $x \in L$ has compact closure. Indeed, it follows from (i) (ii) (iii) that $H$ contains a subgroup $H^{\prime}$ isomorphic to the reals. If $H \neq H^{\prime}$ then $G / H$ would be compact. But if $x \in L$ there is a natural one-to-one continuous map from $G / H$ to $x G$, and this violates (iv).

(i) is obvious from the definition in 3.3. (ii) was proven in 3.3. As to (iii), he only two-dimensional Lie group imbeddable in the plane which has a compact subgroup is the circle cross the line. In this case $L$ has an idempotent and $S$ has a zero. For a complete discussion of this case see [4]. (iv) follows from 2.2.1 and 0.5.

REMARK. From (iii) above we see that $G$ must either be isomorphic to the two-dimensional vector group or to the nonabelian group of affine transformations of the line: $y=a x+b, a>0$.

3.7. Theorem. If the plane $S$ is a semigroup with identity and no other idempotents then it is a group.

Proof. Assume $L$ is not null; we must show that it contains an idempotent. Fix an $x \in L$. If $x^{2} \in x G \cap G x$, then the result follows from 2.4. So we may assume $x^{2} \notin x G$.

Let $R$ be any one-parameter subgroup of $G$ different from $H$. Then $R$ is a cross section for $H$ in $G$. Let $T=\left[a^{-1}, a\right]$ be a symmetric neighborhood of 1 in $R$, and for $x \in L$ let $V$ be a neighborhood of $x^{2}$ of the form $V^{-}=C T$, where $C$ is a local cross section to the local orbits of $T$ at $x^{2}$. Then $V^{-}$is homeomorphic to $C \times T$ under $(c, t) \rightarrow c t$.

$x R$ must cluster at $x^{2}$. Indeed, let $g_{n} \rightarrow x, g_{n} \in G$. Then $x g_{n} \rightarrow x^{2}$. But $x R=x G$, so $x^{2} \in(x R)^{-}$. Hence $x R$ gets inside $V$. We choose the ordering on $R$ 
in which $a>1$. This induces an ordering on $x R$. Let $R_{+}$be the elements $\geqq 1$ in $R$, and $R_{-}$the elements $\leqq 1$. Then either $x R_{+}$or $x R_{-}$clusters at $x^{2}$, so we may assume it is $x R_{+}$.

$C T$ is ruled by the lines $\{c T\}, c \in C$. We call these lines "strips," and give each strip the ordering in which $c a>c$. Since $x^{2} \notin x R$, but $x R_{+}$clusters at $x^{2}$, infinitely many strips must be in $x R_{+}$. From the way we have chosen our orderings, it follows that if the strip $c T$ is in $x R$, then its ordering must agree with the ordering of $x R$.

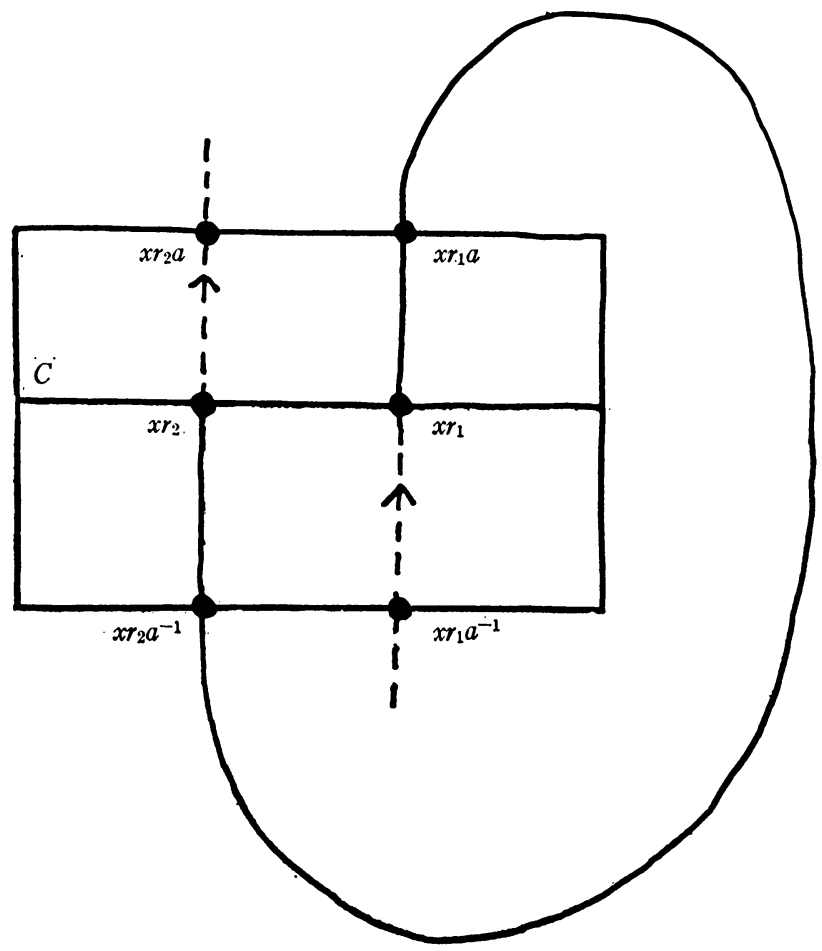

Let $r_{1} \in R_{+}$be such that $x r_{1} \in C$. Then $x r_{1}$ is on the strip $\left[x r_{1} a^{-1}, x r_{1} a\right]$. Let $r_{2}$ be the first element of $R_{+}$greater than $r_{1}$ such that $x r_{2}$ lies on $C$. Then $x r_{2}$ lies on the strip $\left[x r_{2} a^{-1}, x r_{2} a\right]$. Let $D$ denote the simple closed curve consisting of the part of $x R_{+}$from $x r_{1}$ to $x r_{2}$ together with the part of the arc $C$ from $x r_{2}$ to $x r_{1}$. See the figure above where one possible configuration is shown.

$D$ divides the plane into two regions one of which has compact closure. Consider the two points $x r_{1} a^{-1}$ and $x r_{2} a$. One of them is in the region interior to $D$-to fix ideas we assume it is the point $y=x r_{1} a^{-1}$. Then all of $y R_{\text {_ }}$ must be interior to $D$. Indeed, $x R$ is a one-to-one mapping, so $y R_{-}$cannot cross the part of $x R$ between $x r_{1}$ and $x r_{2}$. And if $y R_{-}$were to cross $C$ between $x r_{2}$ and 
$x r_{1}$ it would be on a strip going in the opposite direction to the ordering on the strip, which is impossible.

Therefore $y R_{-}$has compact closure. Let $z$ be a limit point of $y R_{-}$, say $y r_{n} \rightarrow z$. Then $y r_{n} r \rightarrow z r$ for all $r \in R$, and if $n$ is large enough $r_{n} r \in R_{\text {. }}$. Hence $z R$ is contained in the limit points of $y R_{-}$, and therefore has compact closure. By 2.2.1 $(z R)^{-}$is a semigroup and thus contains an idempotent by 0.5 . This completes the proof.

4. With the notations of $\$ 2$ we now assume $S$ is the plane and $G$ is the open right half plane, so that $L$ is the $y$-axis. The problem is to determine the possible multiplications on $L$. These will depend both on the group $G$ and on how $G$ is imbedded in the half plane. There are two possibilities for $G$, it is either isomorphic to the two-dimensional vector group or to the group of affine transformations of the line: $y=a x+b, a>0$. We do not consider the following question. Suppose two such multiplications $G \cup L$ and $G^{\prime} \cup L^{\prime}$ are given, with $G$ isomorphic to $G^{\prime}$ and $L$ isomorphic to $L^{\prime}$. Does it follow that $G \cup L$ is isomorphic to $G^{\prime} \cup L^{\prime}$ ?

As before we work entirely with the semigroup $G^{-}=G \cup L$. Any multiplication in $G^{-}$may be extended to the rest of $S$ in various ways. For example, for $u \in S$ let $p(u)$ be the reflection of $u$ in the $y$-axis. For $u$ and $v$ in the left half plane define $u v=p(p(u) p(v))$. For $u$ in the left half plane and $v$ in the right half plane define $u v=p(p(u) v)$, and $v u=p(v p(u))$.

Any orbit $x G$ that is not a point must be an open interval, finite or infinite.

4.1. $x$ is called a left zero if $x z=x$ for all $z \in G^{-}$. This is equivalent to the right orbit being a point: $x G=x$.

4.1.1. If $x G \neq L$ and $y$ is an endpoint of $x G$, then $y$ is a left zero. Indeed, $y \notin x G$ since $x G$ is an open interval, and $y G$ is a connected subset of $(x G)^{-}$ by 2.2 .2 , so $y G=y$.

4.2. Lemma. If $L$ has a left zero that is not a right zero, then every element in $L$ is a left zero, and there is only one left orbit: $G x=L$.

Proof. By hypothesis there is an element $x \in L$ such that $x G=x$, and $G x \neq x$. Assume $G x \neq L$. Then $G x$ is an open interval with at least one endpoint $y$. Clearly all elements of $G x$ are left zeros, hence $y$ is a left zero too. By the analogue of 4.1.1 $y$ is also a right zero, hence is a two-sided zero. But this is impossible, for then there could be no other left zeros.

4.3. Corollary. If $x G$ has compact closure then it is a point.

Proof. If this were false $x G$ would be an open interval with two endpoints $e$ and $f$. By 4.1 .1 both are left zeros. This means there are no right zeros so by $4.2 x G=x$.

4.4. LeMma. If $L$ does not have a one-sided zero that is not two-sided, then $x G=G x$ for every $x \in L$, and either there are exactly three orbits one of which is a 
zero, or there is only one orbit which is a group.

Proof. Case I. Assume $x G \neq L$ and let $y$ be an endpoint. By 4.1.1 $y$ is a left zero which must be a two sided zero by hypothesis. So $y G=G y=y$. No other left or right orbits can have compact closure by 4.3 so there must be exactly three left orbits and exactly three right orbits. Therefore for $u \in L$ we must have $u G=G u$ since both contain $u$.

CASE II. If $x G=L$, then $G x=L$ by the analogue of Case I for left orbits. By $2.3 L$ is a group.

4.5. THEOREM. If $G$ is abelian then there are exactly four possibilities for the multiplication on $L$ :

I. $L$ is a group.

II. $L$ has a zero, 0 , dividing $L$ into the two sets $A$ and $B$. Then $A B=0$ and we have the three possibilities:

a. $A$ and $B$ are each groups.

b. $A^{2}=B^{2}=0$.

c. $A$ is a group and $B^{2}=0$.

Proof. By 4.4 either (I) $L$ is a group; or (II) $L$ has a zero and there are two other orbits $A$ and $B$. By 2.2.1 $A^{-}$and $B^{-}$are ideals, so $A^{-} B^{-} C A^{-} \cap B^{-}$ $=\{0\}$. By 2.3 there are at most the three possibilities listed for II. The proof will be completed by giving examples of all four possibilities. In the examples the multiplication is given in coordinate form; we omit the verifications. In the second and fourth examples we work in the first quadrant instead of the half plane.

4.5.1. I. $(a, b)(x, y)=(a x, b+y), a, x \geqq 0$. Here $(1,0)$ is the identity.

4.5.2. IIa. $(a, b)(x, y)=(a x, b y), a, b, x, y \geqq 0$. Here $G$ is the open first quadrant and $(1,1)$ is the identity.

4.5.3. IIb. $(a, b)(x, y)=(a x, a y+b x), a, x \geqq 0$. Here $(1,0)$ is the identity.

4.5.4. IIc. Here we work in the first quadrant: $a, b, x, y \geqq 0$. The product is defined in three parts:

$$
\begin{aligned}
& (a, b)(x, y)=\left(a x, b y \frac{1+\log ^{2} a x}{\left(1+\log ^{2} a\right)\left(1+\log ^{2} x\right)}\right) \quad \text { if } a, x>0, \\
& (0, b)(x, y)=\left(0, b y \frac{1}{1+\log ^{2} x}\right) \quad \text { if } x>0, \\
& (0, b)(0, y)=(0,0) .
\end{aligned}
$$

The origin is a zero, $(1,1)$ is the identity, the positive $x$-axis is a group, and the product of any two elements on the positive $y$-axis is zero.

We indicate the verification of the associativity and continuity. Let $\phi(x)$ be continuous and strictly positive for $x>0$, and $\phi(1)=1$. Define a multiplication on the "half-open" first quadrant $a, x>0, b, y \geqq 0$ by: 


$$
(a, b)(x, y)=\left(a x, b y \frac{\phi(a x)}{\phi(a) \phi(x)}\right) .
$$

The associativity is easily checked, $(1,1)$ is an identity, the positive $x$-axis is a group, and the open first quadrant is isomorphic to the two-dimensional vector group. The problem is to choose $\phi$ so that the multiplication may be continuously extended to the $y$-axis in such a way that the product of any two elements on the $y$-axis is zero. If this can be done the associative law will hold automatically since it holds on a dense subset.

Let $f(a, x)=\phi(a x) / \phi(a) \phi(x)$. Then we want:

(i) $f(a, x)$ bounded for $a, x>0$;

(ii) $\lim f(a, x)=0$ as $a, x \rightarrow 0$;

(iii) $\lim f(a, x)$ exists, $>0$, as $a \rightarrow 0$ and $x \rightarrow x_{0}>0$. This limit will necessarily depend continuously on $x_{0}$.

All of these conditions are satisfied if one takes $\phi(x)=1+\log ^{2} x$. The limit in (iii) will then be: $1 /\left(1+\log ^{2} x_{0}\right)$. This completes the proof of Theorem 4.5.

4.5.5. If $S$ is a topological group and $H$ is a subgroup, then $S / H$ is Hausdorff if and only if $H$ is closed. This is not true if $S$ is merely a topological semigroup with identity and $H$ is a closed subgroup containing 1 , even when the cosets $x H$ are closed sets.

For example, let $S$ be the plane with the multiplication of Example 4.5.3, and let $H$ be the vertical line through $(1,0) . H$ is a closed subgroup; the coset containing $(a, b)$ is the vertical line through $(a, b)$ if $a \neq 0$ but is the point $(a, b)$ itself if $a=0$. The coset space $S / H$ is not Hausdorff.

4.6. If $G$ is nonabelian there are at most five possibilities for the multiplication on $L$. These are the four, I, IIa, b, c of Theorem 4.5, and one more, III, in which every element of $L$ is a left zero (or the analogous case where every element is a right zero). This follows from 4.2 and 4.4 just as in the proof of Theorem 4.5. We give examples for IIb and III and we conjecture that the other three cases are not in fact possible.

4.6.1. IIb. $(a, b)(x, y)=\left(a x, a^{2} y+b x\right), a, x \geqq 0$. Here $(1,0)$ is the identity.

4.6.2. III. $(a, b)(x, y)=(a x, a y+b), a, x \geqq 0$. Again $(1,0)$ is the identity. This example is the usual representation of $G$ as the group of matrices

$$
\left(\begin{array}{ll}
a & b \\
0 & 1
\end{array}\right)
$$

In the other three cases, if they exist, one has $x G=G x$ for all $x \in L$, and at least one orbit is isomorphic to the group of real numbers. It can be shown that such ${ }^{\circ}$ an orbit is in the center of $G^{-}$.

\section{BIBLIOGRAPHY}

1. A. D. Wallace, Inverses in Euclidean mobs, Math. J. Okayama Univ. vol. 3 (1953) pp. 23-28. 
2. - Cohomology, dimension and mobs, Summa Brasil. Math. vol. 3 (1953) pp. 43-54.

3. - The structure of topological semigroups, Bull. Amer. Math. Soc. vol. 61 (1955) pp. 95-112.

4. P. S. Mostert and A. L. Shields, On a class of semigroups on $E_{n}$, Proc. Amer. Math. Soc. vol. 7 (1956) pp. 729-734.

TUlane University, New OrLeans. La.

UNIVERSITY OF MiChIGAN, AnN ARbor, Mich. 